\title{
Some Characterization of the Function Space Type of Lizorkin-Triebel-Morrey
}

\author{
Rena Eldar Kizi Kerbalayeva \\ Institute of Mathematics and Mechanics, National Academy Science of Azerbaijan, Baku, Azerbaijan
}

\section{Email address:}

rena-kerbalayeva@mail.ru

\section{To cite this article:}

Rena Eldar Kizi Kerbalayeva. Some Characterization of the Function Space Type of Lizorkin-Triebel-Morrey. Mathematics and Computer Science. Vol. 6, No. 4, 2021, pp. 59-64. doi: 10.11648/j.mcs.20210604.11

Received: May 29, 2021; Accepted: June 8, 2021; Published: August 30, 2021

\begin{abstract}
This paper will introduce you to some properties of normed function spaces with many groups variables field of Analysis and it helps me appreciate how normed Lebesgue-Morrey space with many groups of variables that build and studied new normed spaces nowadays. Many of the topics here are important to an Analysis class. By reading this paper, you will discover the "embedding theory" of normed spaces type of Lebesgue-Morrey by introducing few of its "new functions with groups with variables" and along the way you will see to some interesting and article elements of the branch called Analysis. A lot of problems belonging to the characterization of various spaces of differentiability function spaces and relationships between them have been solved using the theory embedding theorems. The purpose of this paper is to review several embedding inequalities of normed spaces that will arise properties of these spaces and again throughout this material. We also give "working definition, notations" of a functions and function spaces. We must note that, the analysis is based on such function spaces to build new space type of Lizorkin-Triebel-Morrey. In addition, throughout this paper we will introduce a working normed function spaces type of Lizorkin-Triebel-Morrey with standard mathematical definitions and terminology. One aspect of this paper involves normed Lebesgue-Morey type spaces that can convert space from one to another.
\end{abstract}

Keywords: The Space Type of Lesgue-Triebel-Morrey, Function Space of Differentiability Function, Many Groups of Variablesç

\section{Introduction and Main Results}

Let $G \subset R^{n}$ and $1 \leq s \leq n ; s, n$ be naturals, where $n_{1}+\cdots+n_{s}=n$. We consider the sufficient smooth function $\mathrm{f}(\mathrm{x})$, where the point $x=\left(x_{1}, \ldots, x_{s}\right) \in R^{n}$ has coordinates $x_{k}=\left(x_{k .1} ; \ldots ; x_{k, n_{k}}\right) \in R^{n_{k}} \quad\left(\mathrm{k} \in e_{s}=\{1, \ldots, \mathrm{s}\}\right)$. More precisely, $R^{n}=R^{n_{1}} \times R^{n_{2}} \times \cdots \times R^{n_{s}}$. Thus we consider the fixed, non-negative, integral vector $\mathrm{l}=\left(l_{1}, \ldots, l_{s}\right)$ such that, $l_{k}=\left(l_{k .1} ; \ldots ; l_{k, n_{k}}\right), \quad\left(\mathrm{k} \subset e_{s}\right)$ that is, $l_{k, j}>0, \quad(j=$ $\left.1, \ldots, n_{k}\right)$ for all $k \in e_{s}$. Here we consider by $\mathrm{Q}$ the set of vectors $\mathrm{i}=\left(i_{1}, \ldots, i_{s}\right)$ where $\mathrm{i}_{\mathrm{k}}=1,2, \ldots, n_{k}$ for every $k \in e_{s}$. The number of set $\mathrm{Q}$ is equal to: $|Q|=\prod_{k=1}^{S}\left(1+n_{k}\right)$. Therefore, to the vector $\mathrm{i}=\left(i_{1}, \ldots, i_{s}\right) \in Q$, we shall correspond the vector $l^{i}=\left(l_{1}^{i_{1}} ; \ldots ; l_{s}^{i_{s}}\right)$ of the set of non-negative, integral vectors $1=\left(l_{1}, \ldots, l_{s}\right)$, where $l^{0}=$ $(0,0, \ldots, 0), l_{k}^{1}=\left(l_{k, 1}, 0, \ldots, 0\right), \ldots, l_{k}^{i_{k}}=\left(0,0, \ldots, l_{k, n_{k}}\right)$ for all $k \in e_{s}$. Then to the vector $e^{i}$, we let correspond the vector $\vec{l}^{\imath}=\left(\vec{l}_{1}^{i_{1}}, \vec{l}_{1}^{i_{2}}, \ldots, \vec{l}_{1}^{i_{s}}\right)$, where $\vec{l}_{k}^{i_{k}}=\left(\vec{l}_{k, 1}^{i_{1}}, \vec{l}_{k, 2}^{i_{2}}, \ldots, \vec{l}_{k, n_{k}}^{i_{k}}\right)\left(k \in e_{s}\right)$.
Here the largest number $\bar{l}_{k, j}^{i_{k}}$ is less than $l_{k, j}^{i_{k}}$ for all $l_{k, j}^{i_{k}}>0$, when $l_{k, j}^{i_{k}}=0$ then we assume that $\vec{l}_{k, j}^{i_{k}}=0$ for all $k \in e_{s}$. Theremore, we consider $D^{\bar{l}^{i}} f=D_{1}^{\bar{l}_{1}^{i_{1}}} \cdots D_{s}^{\bar{l}_{s}^{i_{s}}} f, D_{k}^{\bar{l}_{k}^{i_{k}}} f=D_{k, 1}^{\bar{l}_{k}^{i_{k}}}$.. $\cdot D_{k, n_{k}}^{\bar{l}_{k}^{i_{k}}} f, G_{t^{\varkappa}}=G \cap I_{t^{\varkappa}}(x), I_{t^{\varkappa}}(x)=I_{t_{1}^{\varkappa_{1}}}\left(x_{1}\right) \times I_{t_{2}^{\varkappa_{2}}}\left(x_{2}\right) \times$. $\cdots \times I_{t_{s}^{\varkappa_{s}}}\left(x_{s}\right), I_{t_{k}^{\varkappa_{k}}}\left(x_{k}\right)=\left\{y_{k}:\left|y_{k}-x_{k}\right|<\frac{1}{2} t_{k}^{\left|\varkappa_{k}\right|}, k \in e_{s}\right\}$ and $\left|\beta_{k}\right|=\sum_{j=1}^{n_{k}} \beta_{k, j}^{i_{k}} ;\left|\beta_{k}^{i_{k}}\right|=\sum_{j=1}^{n_{k}} \beta_{k, j}^{i_{k}} \frac{d t_{k}}{t_{k}}=\prod_{j \in e_{k}^{i} \frac{d t_{k, j}}{t_{k, j}}}$, we take $0<\beta_{k, j}^{i_{k}}=l_{k, j}^{i_{k}}-\bar{l}_{k, j}^{i_{k}} \leq 1$, when $l_{k}^{i_{k}}>0$, but when $l_{k, j}^{i_{k}}=0$, then $\beta_{k, j}^{i_{k}}=0 ; t=\left(t_{1}, \ldots, t_{s}\right)$, $t_{k}=\left(t_{k, 1}, \ldots, t_{k, n_{k}}\right), \omega=\left(\omega_{1}, \ldots, \omega_{s}\right), \omega_{k}=$

$\left(\omega_{k, 1}, \ldots, \omega_{k, n_{k}}\right)$ and we take $\omega_{k, j}=1$, when $\mathrm{k} \in e^{i}$, or we give $\omega_{k, j}=0$, when $\mathrm{k} \in e_{s} / e^{i}, e^{i}=\operatorname{supp} \vec{l}^{i}=\operatorname{suppl}^{i}=$ supp $\omega, \quad 1 \leq \theta \leq \infty ; 1 \leq p<\infty \quad ; \quad$ Here $t_{0}=\left(t_{0,1}, \ldots, t_{0, s}\right), t_{0, k}=\left(t_{0, k, 1}, \ldots, t_{0, k, n_{k}}\right)-$ is fixed vector and $\mathcal{x \in}(0, \infty)^{n}, \mathrm{a} \in[0,1], \tau \in[1, \infty],\left[t_{k}\right]_{1}=\min \left\{1, t_{k}\right\}, k \in$ 
$e_{s} . \quad$ Here $\Delta^{\omega}(t) f=\Delta_{1}^{\omega_{1}}\left(t_{1}\right) \cdots \Delta_{s}^{\omega_{s}}\left(t_{k}\right) f$, when $2 \omega=$ $(2,2, \ldots, 2)$ $\Delta_{k}^{\omega_{k}}\left(t_{k}\right) f=\Delta_{k, 1}^{\omega_{k, 1}}\left(t_{k, 1}\right) \cdots \Delta_{k, n_{k}}^{\omega_{k, n_{k}}}\left(t_{k, n_{k}}\right) f,\left(k \in e_{s}\right)$

following $\Delta_{k, j_{k}}^{\omega_{k, j_{k}}}\left(t_{k, j_{k}}\right) f$ are finite difference function, which has the direction with variables $t_{k, j_{k}}$ and with order $\omega_{k, j_{k}}$, by step $t_{k, j_{k}}$ for $j=1, \ldots, n_{k}$ and for all and $k \in e_{s}$, following

$$
\|f\|_{p, G_{t^{\varkappa}}(x)}=\left\{\int_{G_{t^{\varkappa_{n}}\left(x_{n}\right)}}\left[\cdots\left\{\int_{G_{t^{\varkappa_{2}}\left(x_{2}\right)}}\left(\int_{G_{t^{\varkappa_{1}}\left(x_{1}\right)}}|f(y)|^{p_{1}} d y_{1}\right)^{p_{2} / p_{1}} d y_{2}\right\}^{p_{3} / p_{2} \ldots}\right]^{p_{n} / p_{n-1}} d y_{n}\right\}^{1 / p_{n}}
$$

and $\mathrm{dy}=\prod_{i=1}^{n} d y_{i}$.

It is said, that the subdomain $U \subset G \subset R^{n}$ calls domain satisfying the condition " $\sigma-$ semi-horn", if the vector $\sigma=\left(\sigma_{1}, \ldots, \sigma_{s}\right)$ is such that $x+V(\sigma) \subset G$ for all $x \subset U$. It is said, that the domain $G \subset R^{n}$ calls domain satisfying the condition " $\sigma$-semi-horn", that is, $G \subset A\left(T^{\sigma}\right)$, if we have finite subdomains $G_{1}, \ldots, G_{N} \subset G$, satisfying the condition " $\sigma-$ semi-horn" and the surfacing the domain $\mathrm{G}$, that is,

$$
G \subset \bigcup_{j=1}^{N} G_{j} .
$$

But we suppose $G \in A_{\epsilon}\left(T^{\sigma}\right)$, if we substitute the condition

\section{Definitions and Preliminaries}

Definition 1 . We denote by

$$
F_{p, \theta, a, \varkappa, \tau}^{<l>}(\mathrm{G}, \mathrm{s})(1<\theta<\infty)
$$
with many groups variables, with finite norm
$\Delta_{k, j_{k}}^{1}\left(t_{k, j_{k}}\right) f\left(\cdots, x_{k, j_{k}}, \cdots\right)=f\left(\cdots, x_{k, j_{k}}+t_{k, j_{k}}, \cdots\right)-$ $f\left(\cdots, x_{k, j_{k}}, \cdots\right), \quad$ and $\quad \Delta_{k, j_{k}}^{\omega_{k, j_{k}}}\left(t_{k, j_{k}}\right) f\left(\cdots, x_{k}, j_{k}, \cdots\right)=$ $\Delta_{k, j_{k}}^{1}\left(t_{k, j_{k}}\right)\left\{\Delta_{k, j_{k}}^{\omega_{k, j_{k}}{ }^{-1}}\left(t_{k, j_{k}}\right) f\left(\cdots, x_{k, j_{k}}, \cdots\right)\right\}$, but when $\omega_{k, j_{k}}=0$, then $\Delta_{k, j_{k}}^{0}\left(t_{k, j_{k}}\right) f\left(\cdots, x_{k, j_{k}}, \cdots\right)=f\left(\cdots, x_{k, j_{k}}, \cdots\right.$ ). Here

normed Lizorkin-Triebel-Morrey space of function $f$ on $G$,

$$
\|f\|_{\mathrm{F}_{\mathrm{p}, \theta, \mathrm{a}, \mathcal{u}, \tau}^{<l,}(\mathrm{G}, \mathrm{s})}=\sum_{i \in Q}\|f\|_{L_{p, \theta, a, \varkappa, \tau}^{<i^{i}>}(G ; s)},
$$
where

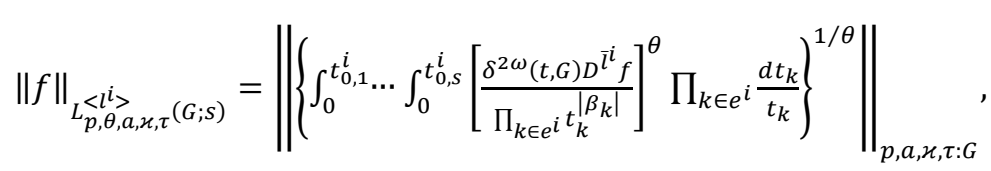

and

$$
\begin{gathered}
\delta^{2 \omega}(t) f(x)=\int_{-1}^{1} \cdots \int_{-1}^{1}\left|\Delta^{2 \omega}\left(t, G_{t}\right) f(x)\right| d t \\
\|f\|_{p, a, \varkappa, \tau: G}=\sup _{x \in G}\left\{\int_{0}^{\infty} \cdots \int_{0}^{\infty}\left[\prod_{k \in e_{s}}\left[t_{k}\right]_{1}^{\frac{-\left|\varkappa_{k}\right| a}{p}} \times\|f\|_{p, G_{t} \varkappa(x)}\right]^{\tau} \prod_{k \in e_{s}} \frac{d t_{k}}{t_{k}}\right\}^{1 / \tau} .
\end{gathered}
$$

Here $G \subset R^{n}, \quad l^{i}, \bar{l}^{i}, p, a, \varkappa, \tau, e^{i}, e_{s}, \beta_{k}$ and $\left[t_{k}\right]_{1}$ are defined in chapter 1 .

We must note that, when $\mathrm{s}=1$, then the space $F_{p, \theta, a, \mathcal{u}, \tau}^{<l>}(s, G)$ is equivalent to the space Lizorkin-Triebel-Morrey $\mathrm{F}_{\mathrm{p}, \theta, a, \mathcal{\ell}, \tau}^{<l>}(\mathrm{G})$, when $\mathrm{s}=\mathrm{n}$, then this space is equivalent to the space Lizorkin-Triebel-Morrey type with dominant mixed

$$
\mathrm{B}_{\mathrm{p}, \theta, \mathrm{a}, \mathcal{u}, \tau}^{<l>}(\mathrm{s}, \mathrm{G})
$$

Besov-Morrey space type of loccaly summable $\mathrm{f}$, on $\mathrm{G}$, with finite norm $(1 \leq \theta \leq \infty)$

$$
\begin{aligned}
& \|f\|_{\mathrm{B}_{\mathrm{p}, \theta, \mathrm{a}, \mathcal{u}, \tau}^{<l>}(\mathrm{s}, \mathrm{G})}=\sum_{i \in Q}\|f\|_{L_{p, \theta, a, \mathcal{u}, \tau}^{<i}(G ; s)}, \\
& \|f\|_{L_{p, \theta, a, \varkappa, \tau}^{<l^{i}>}(G ; s)}=\left\{\int_{0}^{t_{0}} \cdots \int_{0}^{t_{0}}\left\|\frac{\Delta^{2 \omega}(t ; G) D^{\bar{l}^{i}} f}{\prod_{k \in e^{i}} t_{k}^{\left|\beta_{k}\right|}}\right\|_{p, a, \mathcal{\varkappa}, \tau: G}^{\theta} \times \prod_{k \in e^{i}} \frac{d t_{k}}{t_{k}}\right\}^{1 / \theta},
\end{aligned}
$$

and 


$$
\|f\|_{p, a, \varkappa_{,} \tau: G}=\sup _{x \in G}\left\{\int_{0}^{\infty} \cdots \int_{0}^{\infty}\left[\prod_{k \in e_{s}}\left[t_{k}\right]_{1}^{-\frac{\left|\varkappa_{k}\right| a}{p}} \times\|f\|_{p, G_{t} \varkappa(x)}\right]^{\tau} \prod_{k \in e_{s}} \frac{d t_{k}}{t_{k}}\right\}^{1 / \tau},
$$

where $1 \leq p<\infty, a \in[0,1], \varkappa \in(0, \infty)^{n}, 1 \leq \tau \leq \infty,\left[t_{k}\right]_{1}=\min \left\{1, t_{k}\right\}, t_{0}$-fixed, positive number.

When $\theta=\infty$, then the space "(5)" is equivalent to the space type of Nickolski - Morrey:

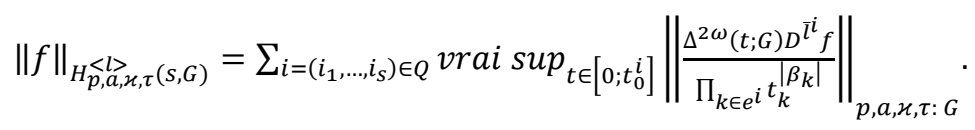

When $\mathrm{s}=1$ then the space "(5)" is equivalent to the space type of $\mathrm{B}_{\mathrm{p}, \theta, \mathrm{a}, \boldsymbol{\alpha}, \tau}^{<l>}(\mathrm{G})$, when $\tau=\infty$ then the space "(5)" is equivalent to the space type $B_{p, \theta, a, \varkappa}^{l}(G)$, when $\mathrm{s}=\mathrm{n}$, then the space "(5)" is equivalent to the space type $S_{\mathrm{p}, \theta, \mathrm{a}, \boldsymbol{u}, \mathrm{\tau}}^{<l>} \mathrm{B}(\mathrm{G})$, when $\mathrm{a}=0, \tau=\infty$, then the space "(5)" is equivalent to the space type $\mathrm{B}_{\mathrm{p}, \theta}^{<l>}(\mathrm{s}, \mathrm{G})$, which was studied by $\mathrm{A} . \mathrm{Dj}$. Djabrailov and in $[4,11,13,15,17]$.

Definition 3. We denote by

$$
\mathrm{W}_{\mathrm{p}, \theta, \mathrm{a}, \mathcal{x}, \tau}^{<l}(\mathrm{~s}, \mathrm{G})
$$

$$
\left\{\int_{0}^{\infty} \cdots \int_{0}^{\infty} \prod_{k \epsilon e_{S}}\left(\left[t_{k}\right]_{1}^{-\frac{\left|\varkappa_{k}\right| a}{p}} \times\|f\|_{p, G_{t} \varkappa(x)}\right)^{\tau} \prod_{k \in e_{s}} \frac{d t_{k}}{t_{k}}\right\}^{1 / \tau} .
$$

Here if $\tau=\infty$, then we get

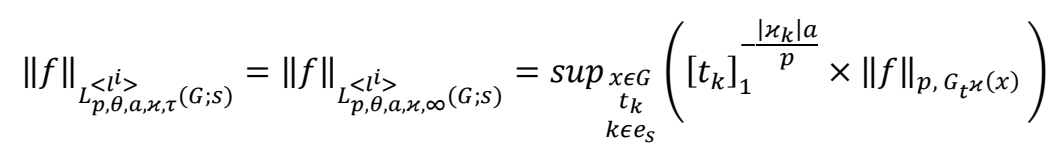

which was studied in $[6,12,19]$.

\section{Proofs of Some Properties}

Let us note some properties of the normed space $F_{\mathrm{p}, \theta, \mathrm{a}, \boldsymbol{x}, \mathrm{\tau}}^{<\mathrm{r}}(\mathrm{s}, \mathrm{G})$ :

1) For any $x>0,1 \leq \tau \leq \infty, c>0$, we obtain

$$
\|f\|_{\mathrm{F}_{\mathrm{p}, \theta}^{<l>}(\mathrm{G}, \mathrm{s})} \leq\|f\|_{\mathrm{F}_{\mathrm{p}, \theta, \mathrm{a}, \mathcal{\chi}}^{<l>}(\mathrm{G}, \mathrm{s})} \leq \mathrm{C}_{2}\|f\|_{\mathrm{F}_{\mathrm{p}, \theta, \mathrm{a}, \mu, \mathrm{\tau}}^{<l>}(\mathrm{G}, \mathrm{s})} .
$$

2) The function space $F_{p, \theta, a, \varkappa, \tau}^{<l>}(s, G)$ is complete. The spaces $L_{p, a, \varkappa, \tau}(G)$ and $F_{\mathrm{p}, \theta}^{\mathrm{l}}(\mathrm{s}, \mathrm{G})$ are complete, that is why the space $F_{\mathrm{p}, \theta, \mathrm{a}, \mathcal{L}, \mathrm{\tau}}^{<\mathrm{l}}(\mathrm{s}, \mathrm{G})$ is complete.

3) For any real number $\mathrm{c}>0$

$$
\|f\|_{\mathrm{F}_{\mathrm{p}, \theta, \mathrm{a}, \mathrm{c}, \boldsymbol{c}, \tau}(\mathrm{G}, \mathrm{s})}=\frac{1}{c^{\bar{\tau}}}\|f\|_{\mathrm{F}_{\mathrm{p}, \theta, \mathrm{a}, \mathrm{x}, \mathrm{\tau}}(\mathrm{G}, \mathrm{s})} .
$$

4) $\|f\|_{\mathrm{F}_{\mathrm{p}, \theta, 0, \chi_{,} \infty}^{<l,(\mathrm{G}, \mathrm{s})}}=\|f\|_{\mathrm{F}_{\mathrm{p}, \theta}^{<l>}(\mathrm{G}, \mathrm{s})}$.

5) If $G$ limited domain and

$$
p \leq q, \frac{1-b}{q} \leq \frac{1-a}{p}, 1 \leq \tau_{1} \leq \tau_{2} \leq \infty
$$

then we have

$$
\mathrm{F}_{\mathrm{q}, \theta, \mathrm{b}, \boldsymbol{x}_{,} \tau_{1}}^{<l>}(G ; s) \subset_{>} \mathrm{F}_{\mathrm{p}, \theta, \mathrm{a}, \boldsymbol{u}, \tau_{2}}^{<l>}(G ; s)
$$

and

$$
\|f\|_{p, a, \varkappa, \tau_{2}: G} \leq\|f\|_{q, b, \varkappa, \tau_{1}: G} .
$$


6) Observe that, $1<\theta \leq r \leq s \leq \sigma<\infty$ and $\theta \leq p \leq \sigma$, then we get

$$
\mathrm{B}_{\mathrm{p}, \theta, \mathrm{a}, \mathcal{u}, \tau}^{<l>}(\mathrm{G}, \mathrm{s}) \subset_{>} \mathrm{F}_{\mathrm{p}, \mathrm{r}, \mathrm{a}, \mathcal{u}, \tau}^{<l>}(\mathrm{G}, \mathrm{s}) \subset_{>} \mathrm{F}_{\mathrm{p}, \mathrm{s}, \mathrm{a}, \mathrm{u}, \tau}^{<l>}(\mathrm{G}, \mathrm{s}) \subset_{>} \mathrm{B}_{\mathrm{p}, \sigma, \mathrm{a}, \mathcal{u}, \tau}^{<l>}(\mathrm{G}, \mathrm{s}) .
$$

If we shall be using some well-known facts in [2] about the about of normed spaces then we shall get following embedding:

$$
\mathrm{B}_{\mathrm{p}, \theta}^{l}(\mathrm{G}) \subset_{>} \mathrm{F}_{\mathrm{p}, \mathrm{r}}^{l}(\mathrm{G}) \subset_{>} \mathrm{B}_{\mathrm{p}, \mathrm{s}}^{l}(\mathrm{G}) \subset_{>} \mathrm{B}_{\mathrm{p}, \sigma}^{l}(\mathrm{G})
$$

If $l \in N^{n}, r=s=2$, then

$$
\mathrm{B}_{\mathrm{p}, \theta, \mathrm{a}, \mathcal{u}, \tau}^{<l>}(\mathrm{G}, \mathrm{s}) \subset_{>} \mathrm{F}_{\mathrm{p}, 2, \mathrm{a}, \mathcal{u}, \tau}^{<l>}(\mathrm{G}, \mathrm{s})=\mathrm{W}_{\mathrm{p}, \mathrm{a}, \mathcal{u}, \tau}^{<l>}(\mathrm{G}, \mathrm{s}) \subset_{>} \mathrm{B}_{\mathrm{p}, \sigma, \mathrm{a}, \mathcal{u}, \tau}^{<l}(\mathrm{G}, \mathrm{s})
$$

In this case when $\mathrm{p}=\theta$, then $\mathrm{F}_{\mathrm{p}, \mathrm{p}, \mathrm{a}, \mathcal{u}, \tau}^{<l>}(\mathrm{G}, \mathrm{s})=\mathrm{B}_{\mathrm{p}, \mathrm{p}, \mathrm{a}, \mathcal{u}, \tau}^{<l,}(\mathrm{G}, \mathrm{s})$.

Here the space $W_{p, a, \varkappa, \tau}^{<l>}(G, s)$ was defined and studied in $[2,8,12,13]$.

We proof first that properties $1,2,3,4,5$ is sufficient for any for any normed $\|f\|_{p, a, \mathcal{\varkappa}, \tau: G}$ [7]. Because of

$$
\|f\|_{p, a, \varkappa, \tau: G}=\sup _{x \in G}\left\{\int_{0}^{\infty} \cdots \int_{0}^{\infty}\left[\prod_{k \in e_{S}}\left[t_{k}\right]_{1}^{-\frac{\left|\varkappa_{k}\right| a}{p}} \times\|f\|_{p, G_{t} \varkappa(x)}\right]^{\tau} \prod_{k \in e_{s}} \frac{d t_{k}}{t_{k}}\right\}^{1 / \tau}
$$

is given same.

Proof 6. Now we must assume that, $1<\theta \leq r \leq s \leq \sigma<\infty$ and $\theta \leq p \leq \sigma$, then we have to proof

$$
\mathrm{B}_{\mathrm{p}, \theta, \mathrm{a}, \mathcal{u}, \tau}^{<l>}(\mathrm{G}, \mathrm{s}) \subset_{>} \mathrm{F}_{\mathrm{p}, \mathrm{r}, \mathrm{a}, \mathcal{u}, \tau}^{<l>}(\mathrm{G}, \mathrm{s}) \subset_{>} \mathrm{F}_{\mathrm{p}, \mathrm{s}, \mathrm{a}, \mathcal{u}, \tau}^{<l>}(\mathrm{G}, \mathrm{s}) \subset_{>} \mathrm{B}_{\mathrm{p}, \sigma, \mathrm{a}, \mathcal{u}, \tau}^{<l>}(\mathrm{G}, \mathrm{s}) .
$$

We know that, if $\mathrm{m}>\mathrm{n}$ and $\mathrm{a}>0$, then $a^{1 / n}<a^{1 / m}$. Then taking $1<\theta \leq r \leq s \leq \sigma<\infty$ and using fifth property of the space $F_{p, \theta, a, \varkappa, \tau}^{<l>}(G, s)$ then we have

$$
\|f\|_{p, \theta, a, \mathcal{\varkappa}, \tau} \leq\|f\|_{p, r, a, \mathcal{\varkappa}, \tau} \leq\|f\|_{p, s, a, \mathcal{\varkappa}, \tau} \leq\|f\|_{p, \sigma, a, \varkappa, \tau} .
$$

Then it follows easy from it

$$
\mathrm{F}_{\mathrm{p}, \sigma, \mathrm{a}, \mathcal{u}, \tau}^{<l>}(\mathrm{G}, \mathrm{s}) \subset_{>} \mathrm{F}_{\mathrm{p}, \mathrm{s}, \mathrm{a}, \mathcal{u}, \tau}^{<l>}(\mathrm{G}, \mathrm{s}) \subset_{>} \mathrm{F}_{\mathrm{p}, \mathrm{r}, \mathrm{a}, \mathcal{u}, \tau}^{<l>}(\mathrm{G}, \mathrm{s}) \subset_{>} \mathrm{F}_{\mathrm{p}, \theta, \mathrm{a}, \mathcal{u}, \tau}^{<l>}(\mathrm{G}, \mathrm{s})
$$

and

$$
\mathrm{B}_{\mathrm{p}, \theta, \mathrm{a}, \mathcal{u}, \tau}^{<l>}(\mathrm{G}, \mathrm{s}) \subset_{>} \mathrm{B}_{\mathrm{p}, \mathrm{r}, \mathrm{a}, \mathcal{u}, \tau}^{<l>}(\mathrm{G}, \mathrm{s}) \subset_{>} \mathrm{B}_{\mathrm{p}, \mathrm{s}, \mathrm{a}, \mathcal{u}, \tau}^{<l>}(\mathrm{G}, \mathrm{s}) \subset_{>} \mathrm{B}_{\mathrm{p}, \sigma, \mathrm{a}, \mathcal{u}, \tau}^{<l>}(\mathrm{G}, \mathrm{s}) .
$$

Taking Minkowski’s inequality we hold

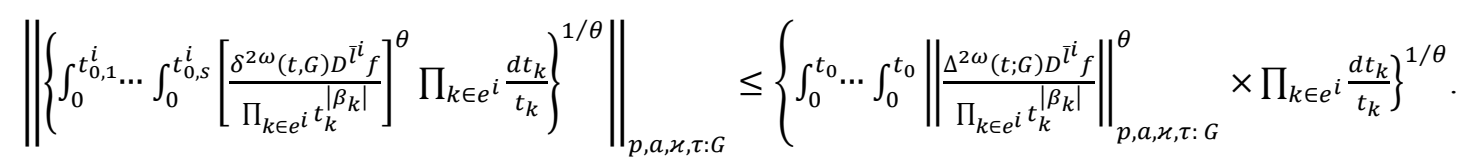

It means that,

$$
\begin{aligned}
& \mathrm{B}_{\mathrm{p}, \sigma, \mathrm{a}, \mathcal{u}, \tau}^{<l>}(\mathrm{G}, \mathrm{s}) \subset \subset_{>} \mathrm{F}_{\mathrm{p}, \sigma, \mathrm{a}, \mathcal{u}, \tau}^{<l>}(\mathrm{G}, \mathrm{s}), \\
& \mathrm{B}_{\mathrm{p}, \theta, \mathrm{a}, \mathcal{u}, \tau}^{<l>}(\mathrm{G}, \mathrm{s}) \subset>\mathrm{F}_{\mathrm{p}, \theta, \mathrm{a}, \mathcal{u}, \tau}^{<l>}(\mathrm{G}, \mathrm{s}) .
\end{aligned}
$$

It is clear that,

$$
\mathrm{B}_{\mathrm{p}, \sigma, \mathrm{a}, \mathcal{u}, \tau}^{<l>}(\mathrm{G}, \mathrm{s}) \subset_{>} \mathrm{F}_{\mathrm{p}, \sigma, \mathrm{a}, \mathcal{u}, \tau}^{<l>}(\mathrm{G}, \mathrm{s}) \subset_{>} \mathrm{F}_{\mathrm{p}, \mathrm{s}, \mathrm{a}, \mathcal{u}, \tau}^{<l>}(\mathrm{G}, \mathrm{s}) \subset_{>} \mathrm{F}_{\mathrm{p}, \mathrm{r}, \mathrm{a}, \mathcal{u}, \tau}^{<l>}(\mathrm{G}, \mathrm{s}) \subset_{>} \mathrm{F}_{\mathrm{p}, \theta, \mathrm{a}, \mathcal{u}, \tau}^{<l>}(\mathrm{G}, \mathrm{s}) .
$$

In addition,

$$
\mathrm{B}_{\mathrm{p}, \sigma, \mathrm{a}, \mathcal{u}, \tau}^{<l>}(\mathrm{G}, \mathrm{s}) \subset_{>} \mathrm{B}_{\mathrm{p}, \sigma, \mathrm{a}, \mathcal{u}, \tau}^{<l>}(\mathrm{G}, \mathrm{s}) \subset_{>} B_{\mathrm{p}, \mathrm{s}, \mathrm{a}, \mathcal{u}, \tau}^{<l>}(\mathrm{G}, \mathrm{s}) \subset_{>} \mathrm{B}_{\mathrm{p}, \mathrm{r}, \mathrm{a}, \mathcal{u}, \tau}^{<l>}(\mathrm{G}, \mathrm{s}) \subset_{>} F_{\mathrm{p}, \theta, \mathrm{a}, \mathcal{u}, \tau}^{<l>}(\mathrm{G}, \mathrm{s}) .
$$

We have proved that

$$
\|f\|_{p, \theta, a, \varkappa, \tau} \leq\|f\|_{p, r, a, \mathcal{\varkappa}, \tau} .
$$


We must note that,

$$
\begin{gathered}
W_{\mathrm{p}, \mathrm{a}, \mathrm{u}, \mathrm{\tau}}^{<l>}(\mathrm{G}, \mathrm{s}) \subset_{>} W_{\mathrm{p}, \mathrm{a}, \mathcal{u}}^{<l>}(\mathrm{G}, \mathrm{s}) \subset_{>} \mathrm{W}_{\mathrm{p}}^{<l>}(\mathrm{G}, \mathrm{s}), \\
B_{\mathrm{p}, \mathrm{a}, \mathcal{u}, \mathrm{\tau}}^{<l>}(\mathrm{G}, \mathrm{s}) \subset_{>} B_{\mathrm{p}, \mathrm{a}, \mathcal{u}}^{<l>}(\mathrm{G}, \mathrm{s}) \subset_{>} \mathrm{B}_{\mathrm{p}}^{<l>}(\mathrm{G}, \mathrm{s}), \\
F_{\mathrm{p}, \mathrm{a}, \mathcal{u}, \mathrm{\tau}}^{<l>}(\mathrm{G}, \mathrm{s}) \subset_{>} F_{\mathrm{p}, \mathrm{a}, \mathcal{u}}^{<l>}(\mathrm{G}, \mathrm{s}) \subset_{>} \mathrm{F}_{\mathrm{p}}^{<l>}(\mathrm{G}, \mathrm{s}),
\end{gathered}
$$

and

$$
\begin{aligned}
& \|f\|_{W_{\mathrm{p}}^{<l>}(\mathrm{G}, \mathrm{s})} \leq\|f\|_{W_{\mathrm{p}, \mathrm{a}, \mathrm{u}}^{<l>}(\mathrm{G}, \mathrm{s})} \leq c\|f\|_{W_{\mathrm{p}, \mathrm{a}, \mu, \mathrm{\tau}}^{<l>}(\mathrm{G}, \mathrm{s})}, \\
& \|f\|_{B_{\mathrm{p}}^{<l>}(\mathrm{G}, \mathrm{s})} \leq\|f\|_{B_{\mathrm{p}, \mathrm{a}, \mathrm{u}}^{<l>}(\mathrm{G}, \mathrm{s})} \leq c\|f\|_{B_{\mathrm{p}, \mathrm{a}, \mathrm{u}, \mathrm{\tau}}^{<l,}(\mathrm{G}, \mathrm{s})}, \\
& \|f\|_{F_{\mathrm{p}}<l>(\mathrm{G}, \mathrm{s})} \leq\|f\|_{F_{\mathrm{p}, \mathrm{a}, \mathrm{u}}^{<l>}(\mathrm{G}, \mathrm{s})} \leq c\|f\|_{F_{\mathrm{p}, \mathrm{a}, \mathrm{u}, \mathrm{\tau}}^{<l>}(\mathrm{G}, \mathrm{s})}
\end{aligned}
$$
[9].

were proved. Having this property for the space $F_{\mathrm{p}, \mathrm{a}, \mathcal{\varkappa}, \tau}^{<l>}(\mathrm{G}, \mathrm{s})$ using fact $F_{\mathrm{p}, \theta, \mathrm{a}, \mathcal{\varkappa}, \tau}^{<l>}(\mathrm{G}, \mathrm{s}) \subset_{>} F_{\mathrm{p}, \theta, \mathrm{a}, \mathcal{\varkappa}}^{<l>}(\mathrm{G}, \mathrm{s})$, one can easy proof it To prove $B_{\mathrm{p}, \theta}^{l}(G) \subset_{>} F_{p, r}^{l}(G) \subset_{>} B_{p, s}^{l}(G) \subset_{>} B_{p, \sigma}^{l}(G)$ we shall use first part of the property 6 .

Now let us proof

$$
\mathrm{B}_{\mathrm{p}, \theta, \mathrm{a}, \mathcal{u}, \tau}^{<l>}(\mathrm{G}, \mathrm{s}) \subset_{>} \mathrm{F}_{\mathrm{p}, 2, \mathrm{a}, \mathcal{u}, \tau}^{<l>}(\mathrm{G}, \mathrm{s})=\mathrm{W}_{\mathrm{p}, \mathrm{a}, \mathcal{u}, \tau}^{<l>}(\mathrm{G}, \mathrm{s}) \subset_{>} \mathrm{B}_{\mathrm{p}, \sigma, \mathrm{a}, \mathcal{u}, \tau}^{<l>}(\mathrm{G}, \mathrm{s}) .
$$

Taking first part of property 6 , we get

$$
\mathrm{B}_{\mathrm{p}, \theta, \mathrm{a}, \mathcal{u}, \tau}^{<l>}(\mathrm{G}, \mathrm{s}) \subset_{>} \mathrm{F}_{\mathrm{p}, 2, \mathrm{a}, \mathcal{u}, \tau}^{<l>}(\mathrm{G}, \mathrm{s})=\mathrm{F}_{\mathrm{p}, 2, \mathcal{u}, \tau}^{<l>}(\mathrm{G}, \mathrm{s}) \subset_{>} \mathrm{B}_{\mathrm{p}, \sigma, \mathrm{a}, \mathcal{u}, \tau}^{<l>}(\mathrm{G}, \mathrm{s})
$$

Then we may replace $\theta=\infty$, then the space "(5)" is equivalent to the space type of Nickolski -Morrey "(8)":

$$
\mathrm{F}_{\mathrm{p}, 2, \mathrm{a}, \mathcal{u}, \tau}^{<l>}(\mathrm{G}, \mathrm{s}) \subset_{>} \mathrm{W}_{\mathrm{p}, \mathrm{a}, \mathcal{u}, \tau}^{<l>}(\mathrm{G}, \mathrm{s})
$$

and

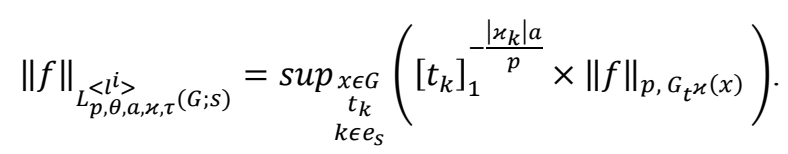

Then we see that a Lebesgue-Triebel-Morrey type space is continuously embedded into a Sobolev-Morrey and there exists a constant $\mathrm{c}$ such as

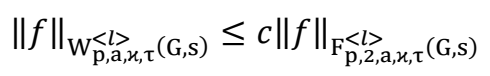

for all $\mathrm{f}$. In addition we get

$$
\mathrm{W}_{\mathrm{p}, \mathrm{a}, \mathcal{u}, \tau}^{<l>}(\mathrm{G}, \mathrm{s}) \subset_{>} \mathrm{F}_{\mathrm{p}, 2, \mathrm{a}, \mathcal{u}, \tau}^{<l>}(\mathrm{G}, \mathrm{s}) .
$$

Then we hold

$$
\mathrm{F}_{\mathrm{p}, 2, \mathrm{a}, \mathcal{u}, \tau}^{<l>}(\mathrm{G}, \mathrm{s})=\mathrm{W}_{\mathrm{p}, \mathrm{a}, \mathcal{u}, \tau}^{<l>}(\mathrm{G}, \mathrm{s})
$$

Taking

$$
\begin{gathered}
\|f\|_{\infty} \leq\|f\|_{1} \leq n\|f\|_{\infty}, \\
\|f\|_{\infty} \leq\|f\|_{2} \leq \sqrt{n}\|f\|_{\infty}, \\
\|f\|_{2} \leq\|f\|_{1} \leq \sqrt{n}\|f\|_{2}
\end{gathered}
$$

Which was studied in $[18,19]$ we can proof property 6 for any $\theta$.

\section{Conclusion}

In this paper I have given and studied normed Lizorkin-Triebel-Morrey type space with many groups of variables. In addition, we can give some properties of these type spaces and some of them were proved. Five of these properties are similar to the already known vanishing properties, but related to the behavior at Lebesgue-TriebelMorrey type spaces instead of that the origin. Another is connected with the Lebesgue-Morrey type to the exterior with many groups of variables. These five additional properties, together with the vanishing property at the origin, allow us that all elements in this new spaces denoted in the sequel by $L_{p, \theta, a, \mathcal{H}, \tau}^{<l^{i}>}(G ; s)$, may be approximated by $B_{p, \theta, a, \mathcal{\varkappa}, \tau}^{<l^{i}>}(G ; s), F_{p, \theta, a, \mathcal{L}, \tau}^{<l^{i}>}(G ; s)$ function spaces in Morrey norm. In this paper, we have done a quick review of some topic that ate absolutely essential to being successful in an Analysis class. We know, that generalized derivatives of the space are dependent of the function, but using integral representation one can see the function is dependent of its generalized derivatives:

$$
\begin{gathered}
f(x)=f_{t} \sigma(x)+\int_{0}^{T} \sum_{i=\left(i_{1}, \ldots, i_{s}\right) \in Q} v^{-1-|\sigma|-t_{i} \sigma_{i}} d v \times \\
\int_{R^{n}} \frac{\delta^{\sigma_{i}} f(x+y)}{\delta_{i}^{\sigma_{i}}} \varphi_{i}\left(v^{-\sigma} y\right) d y, \\
f_{t^{\sigma}}(x)=t^{-|\sigma|} \int_{R^{n}} f(x+y) \varphi_{0}\left(v^{-\sigma} y\right) d y .
\end{gathered}
$$

Present work by the author is devoted to generalizing to 
the concepts of several Lebesgue-Morrey type spaces with many group variables to introduce if more refined results on the well-known inequalities and method integral representation of these type spaces can be obtained and we one obtain relationship between and convert space from one from to another. All spaces are in what we generally consider Lebesgue-Morrey type spaces.

\section{Acknowledgements}

Rena E. Kerbalayeva acknowledgement the support of National Academy Science of Azerbaijan, as well as the hosting support of Professors A. Dj. Djabrailov, A. M. Najafov. The author is grateful to the referees for numerous comments that improved the quality of the paper.

\section{References}

[1] Djabrailov A. Dj. (1993) The method of integral representation in the theory of spaces of function of several groups variables. Kluwer Academic Publishers, 13-79.

[2] Djabrailov A. Dj., Maksudov F. Q. (2000) The method integral representation in the theory of spaces. Baku, 3-190.

[3] Guliyev V. S., Najafov A. M. (2001) The imbedding theorems on the Lizorkin-Triebel-Morrey type space. $3^{\text {rd }}$ International ISAAC Congren. Freie Universitat Berlin, Germany, august, 23-30.

[4] Fan Di., Lu S., Yang D. (1998) Regularity in Morrey spaces of strong solutions to nondivergence elliptic equations with VMO coefficients. Georgian Math. Jour., 5, 425-440.

[5] Guiseppe, S. (1998) Un problema di Darboux in un insieme non limitato. Esistenza, unicita e dipendenza continua della soluzione. Math., 53 (2), 359-373.

[6] Leyla Sh. Kadimova., Kerbalayeva R. E. (2019) On smothness of solution for the higher-order partial equations. Transactions Nat. Acade. Sci. Azerbaijan. Ser. Phys.-Tech. Math. Sci. Mathe., 39 (1), page 83-97.

[7] Kerbalayeva R. E. (2021) Some characterization of the function space type of Lebesgue-Morrey. American Journal of İnformation Science and Technology, 5/12, 25-29.
[8] Kokilashvili V., Samko S. (2003) Singular integrals in weighted Lebesgue spaces with variable exponent. Geor. Math. J., 10 (1), 145-156.

[9] Kozano H. (1994) Comm. Partial Differential Navier-Stokes equations. Yamasaki M. Semilinear heat equations and the Navier-Stokes equation with distributions in new function spaces as initial data, 19, 959-1014.

[10] Lin Tang., Jingshi Xu. (2005) Some properties of Morrey type Besov-Triebel spaces. Math. Nachr., 278 (7/8), 904-917.

[11] Mazzucato, A. I. (2001) Decomposition of Besov-Morrey spaces. Proceedings of Conference on Harmonic Analysis, 215-233.

[12] Najafov, A. M. (2005) On some properties of the function from Sobolev-Morrey type spaces. Central Europen Journal of Mathem., 3 (3), 496-507.

[13] Najafov A. M. (2005) Some properties of functions from the intersection of Besov-Morrey type spaces with dominant mixed derivatives. Proceedings of A. Razmadze Math. Inst., $139,71-82$.

[14] Najafov A. M. (2005) Problem on smoothness of solution of one class hypoelliptic equations. Proceedings of A. Razmadze Math. Inst., 140, 131-139.

[15] Najafov A. M., Kerbalayeva R. E. (2015) Interpolation theorems for spaces Besov-Morrey type. Journal presented by institute Mathematics and Computer Sciences at TskhumAbkhazian Academy of Sciences, Tskhum-Abkhazian, IX-X, 198-212.

[16] Najafov A. M., Kerbalayeva R. E. (2016) Some characterization of functions from Lizorkin-Triebel-Morrey type spaces with many groups variable. Transacions of NAS of Azerbaijan, Issue Mathematics, Series of PhysicalTechnical and Mathematical Sciences, v. 36 (1) 1, 100-111.

[17] Najafov A. M., Kerbalayeva R. E. (2019) The embedding theorems for Besov-Morrey spaces of many groups of variables. Proceedings of A. Razmadze Institute Mathematics. Georgian Academy of Sciences, 26 (1), 125-131.

[18] Perez C. (1991) Weighted norm inequality for general maximal operators. Publicacions Mathe., 35 (1), 169-186.

[19] Taylor, M. (1994/1995) Microlocal analysis on Morrey spaces. Singularities and oscullations (Minneapolis). IMA 91, Math. Appl., Springer, New Yor., 97-135. 